\title{
COMMENTARY
}

\section{Competing interests declared: early interventions and long-term psychological outcomes}

\author{
Alastair M Hull ${ }^{* 1}$ and Janice Rattray ${ }^{2}$ \\ See related research by Usuki et al., http://ccforum.com/content/16/5/R196
}

\begin{abstract}
Survivors of motor vehicle accidents and/or survivors of critical care unit admission are at increased risk of developing post-traumatic reactions such as post-traumatic stress disorder, depression and anxiety. Examining the possible risk factors for the development of these disorders must consider pre-traumatic, peri-traumatic and post-traumatic factors and must do so across domains relating to the trauma, the person and their circumstances. The present study has found propofol administration in the first 72 hours post motor vehicle accident to confer a higher risk for full or partial post-traumatic stress disorder at 6 months. This study highlights concerns that treatment needed acutely post injury may impact adversely on long-term outcome, albeit in a different domain - the psychological.
\end{abstract}

\section{Introduction}

Surviving intensive care is only the start of a journey to recovery for patients with various physical, psychological, pragmatic, social and interpersonal challenges testing their resilience, determination and recovery. The impact ripples onwards to challenge their families and carers in a multitude of ways. From a background of increasing interest in the psychological and quality-of-life outcomes for critical care patients, Usuki and colleagues have examined the potential impact of propofol on a cohort of individuals immediately after motor vehicle accidents [1].

This study touches on a number of important issues, five of which will be discussed in this commentary: the study of the critically ill motor vehicle accident survivor; the transfer of bench science to the clinical setting; the

*Correspondence: ahull@nhs.net

'Multidisciplinary Adult Psychotherapy Service, Old Maternity Building, Perth Royal Infirmary, Perth PH1 1NX, UK

Full list of author information is available at the end of the article inherent difficulties of identifying risk factors; the challenge of targeting interventions to only those patients who need them; and the impact of immediate care on long-term psychological outcome.

\section{Including the critically ill patient}

In a study on motor vehicle accident survivors it is vital to actually include the most severely injured. These patients are often excluded from empirical psychotraumatology studies due to the severity of their illness, with estimates of psychomorbidity underestimated as a result. Whilst the patient's perception of injury severity is important in predicting outcome, and not the illness severity scores, few patients with critical illness are left in any doubt that their injury or illness is significant.

The difficulties of recruiting and retaining participants in critical care studies are reflected in this study, with only $35 \%$ of those completing baseline retained in the study by 6 months. There are a number of potential physical and practical explanations for this factor, but avoidance is a core symptom of post-traumatic stress disorder (PTSD), and thus the true incidence of PTSD post critical care unit $(\mathrm{CCU})$ admission may be obscured by this behaviour.

\section{Application of science to clinical settings}

An inherent weakness in some approaches to treatment for psychological disorders is the absence of a clear scientific rationale and/or underpinning conceptualisation that supports the intervention. One current example of this appears to be the use of diaries for patients in CCUs. Modifications to treatment protocols should demand that empirical findings underpin any proposed changes.

Usuki and colleagues based their study on animal studies reporting the enhancement of fear memory in rats by propofol [2] and the hypothesis that modulating the consolidation of fear memory would be a potential strategy to prevent PTSD developing. Much of our understanding of fear comes from classical conditioning animal studies. Both clinicians and researchers have long understood that conditioned fear may be reinstated after 
apparently successful extinction through therapy (or new contextual learning in the laboratory), so limiting the consolidation of fear memory is an attractive approach if and when possible. If propofol consolidates fear memory, this might be one factor explaining the high rates of posttraumatic reactions.

\section{Risk factors and resilience}

Key foci for the treatment of critical illness are to maximise survival and to protect individuals from further threat. Usuki and colleagues identified a potential risk factor for the development of PTSD [1]. The challenge in this area of research is the number of potential mediators and moderators that may account for their finding. An intriguing question arises: should propofol not be used for any patient or is its prescription a problem only for those already at risk of developing PTSD? Do its positive effects outweigh this suggested potentially serious adverse effect? From a mental health perspective, if it is possible to prevent acute stress disorder or PTSD this is an option worth considering. Many patients clearly also benefit from propofol without developing PTSD.

Turning the issue on its head; will any group of critical care patients suffer for not having propofol treatment, especially those not at risk of PTSD? The traumatic event itself is not enough to cause PTSD, with more than one risk factor required. Whether risk factors act accumulatively or some load for the occurrences of others is unclear. Crucially most CCU-exposed individuals do not develop psychological disorder; chronic maladjustment is not the rule. Resilience, a positive adaptation under stress, is a relatively common phenomenon and can be seen as a dynamic concept with interaction of experiences, individual characteristics and interpersonal relationships; some or all of which may crucially be amenable to change [3].

\section{Targeting interventions to only those needing them}

No traumatic event (including CCU admission) causes psychological illness in everyone experiencing it. Some individuals may have a brief period of distress and lower function but are resilient and quickly return to their previous level of psychological functioning, while others will be resistant and show no or minimal distress. Of those experiencing more significant psychological distress and symptoms, many will recover without intervention and watchful waiting should be employed.

One of the most difficult issues for the long-term psychological care of the CCU survivor is the widespread opinion that features such as chronic physical illness or chronic pain are contraindications (or at least limitations) for psychotherapy. Indeed, psychological intervention post trauma is rarely neutral, and can be psychonoxious, with the most frequently quoted example being singlesession debriefing [4]. The PRaCTICaL study demonstrated that there was no adverse impact of psychological intervention post $\mathrm{CCU}$ admission; post-CCU patients can complete therapy [5]. Clearly, it is vital to target any intervention at those patients needing it.

\section{Impact of immediate care on long-term psychological outcome}

Usuki and colleagues' study has raised the issue of immediate care for individuals in the CCU post trauma impacting adversely upon their long-term psychological outcome. Both a PTSD diagnosis and increased total symptoms were adversely affected by propofol administration within the first 72 hours. Clinicians aim to do no harm but some treatments are both required and distressing; the narratives of patients being treated for burn injuries would attest to this. The example of debriefing noted above demonstrates the impact of an acute psychological intervention causing harm despite the best efforts of the clinicians involved.

\section{Conclusion}

The authors acknowledge some very reasonable limitations of their study, and certainly no study answers all of the questions. However, they have conducted a theoretically-based study on an important group of patients often neglected in trauma research. The authors have considered issues concerning the treatment of the critically ill and its potential outcomes. The study has a number of strengths, the use of the Clinician Administered PTSD Scale [6] diagnostic interview being one; this is the gold standard interview for confirming PTSD diagnosis. Most critical care psychological studies have used self-report measures, which represents a major advance. The Clinician Administered PTSD Scale has a number of scoring rules [7] that would add further weight to the findings noted by Usuki and colleagues.

\section{Abbreviations}

CCU, critical care unit; PTSD, post-traumatic stress disorder.

\section{Competing interests}

The authors declare that they have no competing interests.

\section{Author details}

'Multidisciplinary Adult Psychotherapy Service, Old Maternity Building, Perth Royal Infirmary, Perth PH1 1NX, UK. ${ }^{2}$ School of Nursing and Midwifery, University of Dundee, 11 Airlie Place, Dundee DD1 4HJ, UK.

\section{Published: 31 January 2013}

\section{References}

1. Usuki M, Matsuoka Y, Nishi D, Yonemoto N, Matsumura K, Otomo Y, Kim Y, Kanba S: Potential impact of propofol immediately after motor vehicle accident on later symptoms of posttraumatic stress disorder at 6-month follow up: a retrospective study. Crit Care 2012, 16:R196.

2. Hauer D, Ratano P, Morena M, Scacianoce S, Briegel I, Palmery M, Cuomo V, Roozendaal B, Schelling G, Campolongo R: Propofol enhances memory 
formation via an interaction with the endocannabinoid system. Anaesthesiology 2011, 114:1380-1388.

3. North Atlantic Treaty Organisation: Psychosocial Care for People Affected by Disasters and Major Incidents: A Model for Designing, Delivering and Managing Psychosocial Services for People Involved in Major Incidents, Conflict, Disasters and Terrorism. EAPC(JMC)N(2008)0038. Brussels: NATO; 2008.

4. Wessely S, Rose S, Bisson J: Brief Psychological Interventions ('Debriefing') for Immediate Trauma Related Symptoms and the Prevention of Post Traumatic Stress Disorder. Cochrane Review, The Cochrane Library. Oxford: Update Software; 1999:4.

5. Cuthbertson BH, Rattray J, Campbell MK, Gager M, Roughton S, Smith A, Hull AM, Breeman S, Norrie J, Jenkinson D, Hernández R, Johnston M, Wilson E, Waldmann C: A pragmatic randomised, controlled trial of nurse led intensive care follow up programmes in improving longer-term outcomes from critical illness. The PRaCTICaL study. Br Med J 2009, 339:b3723. doi: 10.1136/bmj.b3723.

6. Blake DD, Weathers FW, Nagy LM, Kaloupek DG, Gusman FD, Charney DS: The development of a clinician-administered PTSD scale. J Traumatic Stress 1995, 8:75-90

7. Weathers FW, Ruscio AM, Keane TM: Psychometric properties of nine scoring rules for the Clinician-Administered Post-traumatic Stress Disorder Scale. Psychol Assess 1999, 11:124-133.

\section{doi:10.1186/cc11916}

Cite this article as: Hull AM, Rattray J: Competing interests declared: early interventions and long-term psychological outcomes. Critical Care 2013,

17:111. 